\title{
Tagungsbericht
}

Arndt Mecke*

\section{PATINFO 2017 - „Europäische Schutzrechtssysteme im Wandel“}

\author{
Ein Tagungsbericht der PATINFO 2017, dem 39. Kolloquium der Technischen Universität \\ Ilmenau über Patentinformation und gewerblichen Rechtsschutz, vom 31. Mai bis zum 2. Juni \\ 2017 in der Festhalle Ilmenau
}

https://doi.org/10.1515/iwp-2017-0054

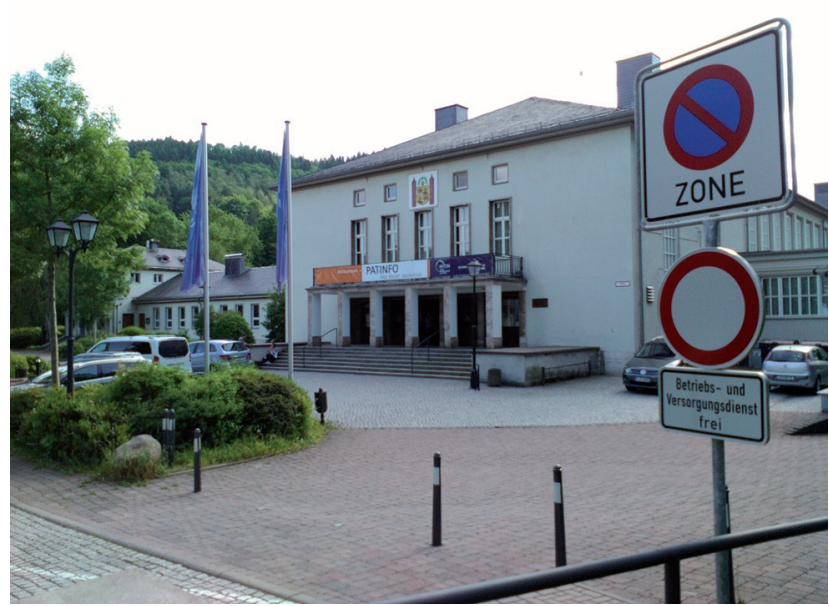

Abbildung 1: Festhalle Ilmenau (Bild: Arndt Mecke).

Einen Tagungsbericht über die PATINFO $2017 \mathrm{zu}$ verfassen, ist nach einer zehnjährigen Abstinenz nicht nur eine persönliche Herausforderung. Denn allen Referenten und ihren zwanzigminütigen Vorträgen mit wenigen Sätzen gerecht zu werden, kann höchstens dazu einladen, sich an Hand des Tagungsbands weiter mit den angerissenen Themen zu beschäftigen.

Die Vorträge des Kolloquiums fanden am 1. und 2. Juni 2017 in der Festhalle in Ilmenau statt. Dazu gab es ein Vorprogramm am 31. Mai 2017 mit kostenfreien Workshops und ein Treffen der PING-Mitglieder („Patentinformationsnutzergruppe“) im PATON. Dieses war leider entgegen meiner Erwartung nicht besonders stark besucht, obwohl hier das weitere Vorgehen im Rahmen der CEPIUG („Confederacy of European Patent Information User Groups“) besprochen und beschlossen werden sollte. PING käme hierbei eine wichtige Aufgabe zur Interessensvertretung der

*Kontaktperson: Arndt Mecke, München, E-Mail: amecke@loop.de deutschsprachigen Patentinformationsnutzer zu. Sowohl in Fragen der Kooperation mit nationalen, regionalen und internationalen Patentämtern als auch beispielsweise im Rahmen der Zertifizierung von Patentrechercheuren als „QPIPs“ (http://www.qpip.org), die in diesem Jahr die letzten Hürden nehmen soll. Da die PATINFO die deutschsprachige Veranstaltung zum Thema Patentinformation ist, würde man sich hier zukünftig eine regere Teilnahme wünschen.

Über ein mangeldes Interesse bei den Besuchern konnte sich die PATINFO 2017 selbst nicht beklagen. Sie zog mehr als 350 angemeldete Kolloquiumsteilnehmer und 43 Aussteller auf zusätzlichen Flächen an.

Über das sehr gut strukturierte Programm möchte ich im Folgenden einen kurzen, chronologischen Überblick geben:

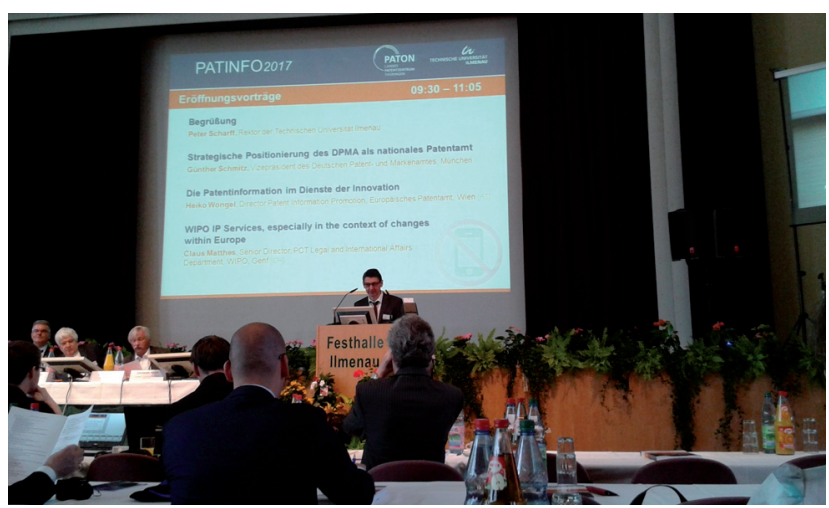

Abbildung 2: Begrüßung durch Dr. Christoph Hoock, Leiter des PATON Patenzentrums Thüringen an der TU Ilmenau (Bild: Arndt Mecke).

Der erste Tag wurde von Ausstellerkurzpräsentationen eingeläutet. Für die Begrüßung bat Christoph Hoock (PATON, Ilmenau) anschließend den Rektor der Technischen Universität Ilmenau Professor Peter Scharff auf das Podium. Dieser ging in seiner Rede auf die Bedeutung des PATON für die TU Ilmenau ein, das eine wichtige Rolle beim 
Schutz und der Verwertung der Forschungsergebnisse spielt. Mit Blick auf den Veranstaltungsort ergänzte er, dass die 40. PATINFO 2018 noch in Ilmenau stattfinden wird, aber für 2019 ein Ausweichquartier gesucht werden muss, damit die Festhalle einer baulichen und technischen Renovierung unterzogen werden kann. Mit Bezug auf das Motto „Europäische Schutzrechtssysteme im Wandel“ sprach er darüber, wie das Europäische Patent mit einheitlicher Wirkung und die Markenrechtsrichtlinie ihre Schatten vorauswerfen. Dieser Wandel bedeute auch hohe und weiter steigende Kosten für die Kleinen und Mittleren Unternehmen (KMU) aber auch für die Universitäten. Da aber Patente von Universitäten auch eine Form von Technologietransfer an die Gesellschaft darstellen, sei dies auch eine Aufgabe für die Hochschulleiter.

Als Einleitung in das Thema „Europäische Schutzrechtssysteme im Wandel" hatte sich das Organisationsteam für Präsentationen von drei Patentämtern entschieden. Den Anfang machte dabei das Deutsche Patent- und Markenamt (DPMA), vertreten durch Günther Schmitz (DPMA, München). In seiner Einleitung hob er hervor, dass die PATINFO ein fest gesetzter Termin im Kalender des DPMA ist. In Bezug auf den Wandel in den Schutzrechtssystemen unterstrich er die Bedeutung der nationalen Patentämter, auch wenn er das Europäische Patent mit einheitlicher Wirkung als wichtigen Schritt ansieht. Entscheidende Kriterien sind hier für die Anmelder die Kosten und die hohe Qualität. Ein Wandel findet aber auch im DMPA statt - so ist ein neues Leitbild zusammen mit einem „Strategiehaus“ in Arbeit. Außerdem soll es mehr Kommunikation mit den Kunden beispielsweise im Rahmen eines Beirats geben. Aber auch Qualitätssicherungsprozesse und internationale Kooperationen und Verfahrensstandardisierungen stehen beim DPMA auf der Agenda. In Bezug auf die politischen Rahmenbedingungen wünschte sich Günther Schmitz ein klareres Bekenntnis der Politik, die durch die Bereitstellung von Prüferstellen aktiv die Arbeit des DPMA unterstützen und eine gewisse Wertschätzung zum Ausdruck bringen könnte.

Für das Europäische Patentamt (EPA) stellte Heiko Wongel (EPA, Wien) eine Studie zur Nutzung von Patentinformation durch Innovatoren (Erfinder) vor. Diese sehr systematische Studie konnte leider nur eine geringe Reichweite erzielen, kommt aber nummerisch auch auf Eckdaten die man selbst vielleicht bisher nur als „Bauchgefühl“ hatte. Der Studie zufolge nutzen immerhin 70 Prozent der Innovatoren Patentinformation, mit den Schwerpunkten technische Information und Wettbewerberbeobachtung, in ihrer täglichen Arbeit. Das EPA kommt daher zu dem Schluss, dass zur Verbesserung der Situation ein einfacherer Zugang und ein breiteres Bewusstsein in der Gesell- schaft für die Bedeutung von Patentinformation gefördert werden sollte. Dazu ist auch eine Steigerung der Qualität und Rechtzeitigkeit der Patentinformation notwendig.

Claus Matthes (WIPO, Genf) ging in seiner Präsentation auf alle Formen des gewerblichen Rechtsschutzes der Weltorganisation für geistiges Eigentum (WIPO) ein, die Patente, Designs und Marken umfassen. Der Zeitenwandel lässt sich aus ihrer Sicht besonders an den Zuwachsraten von Schutzrechtsanmeldungen aus China bei der WIPO festmachen. Bei Patenten stieg die Zahl im vergangenen Jahr um 45 Prozent, bei Marken war sogar einen Zuwachs von 68 Prozent zu verzeichnen. In Bezug auf die Wünsche von Hochschulen bemerkte Claus Matthes, dass es bereits eine sehr kontrovers geführte Diskussion um Gebührenermäßigungen für Hochschulen gibt.

In der folgenden von Rolf W. Einsele (Meissner Bolte, München) geleiteten Expertenrunde kamen sowohl praxisrelevante, aktuelle Änderungen im Markenrecht, strukturelle Änderungen und deren Auswirkungen in einem Industriekonzern als auch möglicherweise eintretende Änderungen an europäischen Rechtssystemen zur Sprache.

Konkret ging Susann Reinhardt (engel patentanwaltskanzlei, Suhl) auf das neue europäische Markenrecht, die veränderte Klassifizierungspraxis und die Erweiterung des Markenbegriffs ein.

Stephan Wolke (ThyssenKrupp Intellectual Property, Essen) beschrieb, mit welchen Konzepten und Strategien er ThyssenKrupp aus einem 100-jährigen „Patentschlaf“ geweckt hat und fit für die Anforderungen der Patentwelt des 21. Jahrhunderts machen will.

Mit einem Blick in die Zukunft führte Benjamin Grau (Murgitroyd, München) in seiner Präsentation das Publikum durch verschiedene Szenarien eines „Brexits“ und die jeweiligen Auswirkungen auf die IP- und Rechtssysteme in Europa.

Auch Peter Kallas (BASF, Ludwigshafen) konnte sich voll auf die gesammelte Expertise seiner Fachleute stützen und sprach über ein Themenspektrum, das von Klassifikationssystemen in Japan über Defensivpublikationen ${ }^{1}$ bis zur Bewertung von Patentmanagementsystemen reichte.

In ihrer Präsentation stellte Jutta Haußer (EPA, Wien) die Grundzüge der japanischen FI-/F-Term Systematik dar, ging aber auch speziell auf das Konzept der Facetten (Facet) und breiten Facetten (Broad Facet) als ergänzendes Rechercheinstrument ein. Dies ist wichtig, da auch heute

1 Defensivpublikationen sind Veröffentlichungen zum Stand der Technik, die Ergebnisse der eigenen FuE publik machen. Sie können eine strategische Alternative sein, wenn Dritte zeitgleich an ähnlichen Entwicklungen arbeiten (Anm.d.Red.). 
noch zwei Drittel der japanischen Publikationen keine Familienmitglieder in einer westlichen Sprache haben.

Paul Rosenich (Patentbüro Paul Rosenich, Triesenberg, Lichtenstein) informierte die Zuhörer über Vor- und Nachteile von Defensivpublikationen und potentielle Einsatzgebiete als Ersatz oder Ergänzung zu konventionellen Patenten oder Gebrauchsmustern.

In einem holistischen Ansatz den Reifegrad eines Patentmanagementsystems zu ermittelt und dessen Weiterentwicklung in der jeweiligen Organisation zu unterstützen, hat sich das von Lothar Walter, Michael Wustmans und Martin G. Möhrle (alle Universität Bremen, Bremen) entwickelte und im Vortrag vorgestellte Modell zur Aufgabe gemacht.

Die letzte Runde von Vorträgen des ersten Tages wurde von Hubert Rothe (DPMA, München) moderiert und beschäftigte sich mit Fragestellungen rund um Technologietransfer aus verschiedenen Blickwinkeln.

Ausgehend von grundsätzlichen Überlegungen zur Wissensökonomie betrachtete Roya Ghafele (Oxfirst, Oxford, Vereinigtes Königreich) konkrete Ansätze für die Evaluierung des Erfolgs von Technologietransfer und den entsprechenden Strukturen, die im angelsächsischen Raum anders ausgeprägt sind als in Kontinentaleuropa. Basierend auf dieser Bewertung empfiehlt sie den Technologietransfer, speziell im deutschsprachigen Raum, noch mehr zu fördern.

Der Vortrag von Axel Koch (Universität des Saarlandes, Saarbrücken) griff diesen Gedanken auf und erläuterte den Ansatz der Universität des Saarlandes, mit welchen speziellen Strukturen dort Erfolge erzielt wurden. Auch er kam zu dem Ergebnis, dass ein grundlegender Strukturwandel bei den Hochschulen und den Entscheidungsträgern notwendig ist, um dem Technologietransfer auf breiterer Front zum Erfolg zu verhelfen.

Bruno Götz (TÜV Rheinland, Nürnberg) beschäftigte sich in seiner Präsentation mit einem Verfahren zu Bewertung von Patenten und Gebrauchsmustern für eine IPStrategie im Unternehmen. Im vorgestellten Ansatz werden für das Unternehmen spezifische - aber objektive und reproduzierbare - Kenngrößen ermittelt, die nachvollziehbare Entscheidungen ermöglichen.

Im Anschluss hatten die Aussteller erneut Gelegenheit ihre Produkte in Kurzpräsentationen vorzustellen.

Um die Zeit bis zur Abendveranstaltung, die den ersten Vortragstag ausklingen ließ, zu überbrücken, boten die Organisatoren einen Spaziergang zur St.-Jakobus-Kirche mit anschließendem Konzert an.

Sabine Milde (PATON, Ilmenau) moderierte den ersten Themenblock des zweiten Tages, der ganz im Zeichen von Marken- und Designrecht stand.
Die speziellen Punkte, die bei der Umsetzung der EUMarkenrechtsrichtlinie zu beachten sind und die konkret geplanten Umsetzungen ins deutsche Recht, stellte Marius Leber (Bundesministerium der Justiz und für Verbraucherschutz, Berlin) vor. Hauptpunkte waren die nicht mehr erforderliche grafische Darstellbarkeit und die Einführung einer Gewährleistungsmarke.

Claudia Meindel (Euro-Triumph, München) sprach anschließend über die mehr als hundertjährige Praxis im internationalen und regionalen Markenrecht im Unternehmen Triumph International. Im ersten Teil stellte sie dabei die Vor- und Nachteile verschiedener Markenschutz-Systeme vor, während sie im zweiten Teil auf praktische Aspekte in Deutschland und Europa einging.

Designschutz als eingetragenes Design (bisher Geschmacksmuster) erscheint deutlich konkreter als z.B. der Schutzumfang eines Patents, doch wie Henning Hartwig (Bardehle Pagenberg, München) anhand von Beispielen aus der aktuellen Rechtspraxis zeigte, gibt es auch hier einen Interpretationsspielraum der Gerichte.

Markus Ortlieb (DPMA, Jena) erläuterte die verschieden Möglichkeiten und Grundlagen für das seit 31/2 Jahren bestehende Nichtigkeitsverfahren für beim DPMA eingetragene Designs und informierte über die Erfahrungen des DPMA mit dem neuen Instrument.

Der letzte, aber bestimmt nicht uninteressanteste, Teil der Veranstaltung wurde von Elke Thomä (PATON, Ilmenau) geleitet und beschäftige sich mit verschiedenen Rechercheansätzen und den hierfür verfügbaren Werkzeugen.

Den Anfang machte Susanne Köhler (Meissner Bolte, München) mit Ihrem Vortrag über FTO (Freedom-To-Operate) Recherchen und dem Spannungsfeld zwischen den beteiligten Akteuren sowie den Unsicherheiten bei diesem Recherchetyp, die selbst bei optimaler Kooperation von Auftraggeber, Patentanwalt und Rechercheur unvermeidlich sind.

Claus Matthes (WIPO, Genf) durfte als Vertretung ein zweites Mal auf das Podium und stellte neue Funktionen von PATENTSCOPE, dem Recherchetool der WIPO, vor. Er hob dabei speziell die Werkzeuge zur Suche in fremdsprachigen Dokumenten und die verbesserten Übersetzungsmöglichkeiten sowie die neue Suchfunktion für chemische Verbindungen hervor.

Einen unkonventionellen Ansatz zur Nutzung der sprachlichen Strukturen in Patenten präsentierte Yvonne Wich (Fraunhofer Institut für Arbeitswirtschaft und Organisation IAO, Stuttgart). Die vorgestellte Lösung erzeugt durch linguistische Analyse Matrizen von publizierten Aufgaben- und Lösungspaaren, die dann ausgewertet und beispielsweise mit Hilfe von TRIZ-Methoden auf mögliche 
aber noch nicht realisierte Lösungsmöglichkeiten durchsucht werden.

Der abschließende Vortrag von Gabriele Kirch-Verfuß (WissensWert Unternehmensberatung, Recklinghausen) beschäftigte sich mit einem Vergleich der klassischen Patentrecherche (Stichworte, Synonyme, Klassifikation, ...) mit verschieden „intelligenten“ (semantischen) Recherchewerkzeugen. Speziell die umfassende, systematische und schrittweise Aufbereitung des gewählten Beispiels und der jeweiligen Daten für die beiden Rechercheansätze könnten ein hervorragender Ausgangspunkt für weitere ähnliche Analysen in anderen Technologiegebieten und an anderen Beispielen sein.

Die Verabschiedung durch Christoph Hook (PATON, Ilmenau) war gleichzeitig auch die Einladung zur 40. PATINFO, die vom 13. bis 15. Juni 2018 in Ilmenau stattfinden wird.

Zusammenfassend möchte ich feststellen, dass die PATINFO nichts an Attraktivität eingebüßt hat. Bei der ausgewogenen Themenauswahl und mit Blick auf die eingeladenen Experten waren, trotz persönlicher Schwerpunkte, alle Vorträge interessant. Da Veranstaltungen bei uns im Wechsel besucht werden, wird leider wieder einige
Zeit vergehen, bis ich wieder an einer PATINFO teilnehmen kann. Ich wünsche den Organisatoren weiterhin ein glückliches Händchen bei der Auswahl der Themen und Referenten!

Deskriptoren: Tagung, Patent, Patentwesen, Patentklassifikation, Europa, Japan

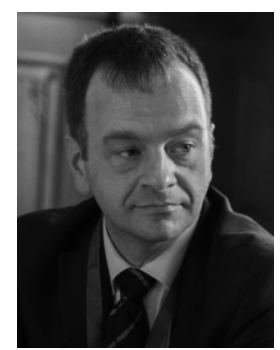

\section{Arndt Mecke}

München

amecke@loop.de

Arndt Mecke arbeitet seit 2001 in der Abteilung Patentinformation eines großen deutschen Elektrokonzerns, nachdem er verschiede ITPositionen bei mittelständischen Unternehmen innehatte. Er betreut mit seinen Kollegen ein firmeneigenes Patent-Datenbanksystem, das allen Mitarbeitern des Unternehmens weltweit zur Verfügung steht. Arndt Mecke ist auch in der PDG Arbeitsgruppe IMPACT aktiv. 